\title{
A Biologic Surgical Implant in Complex Abdominal Wall Repair: 3-Year Follow-Up Results of a Multicentric Prospective Study
}

\author{
Francesco Gossetti ${ }^{1}$ \\ Nikolaus Zuegel $\mathbb{D D}^{2}$ \\ Pasquale Giordano 3 \\ Rupert Pullan ${ }^{4}$ \\ Jochen Schuld ${ }^{5}$ \\ Paolo Delrio ${ }^{6}$ \\ Marco Montorsi ${ }^{7}$ \\ Olivier van Kerschaver ${ }^{8}$ \\ Jean Lemaitre ${ }^{9}$ \\ Ben Griffiths ${ }^{10}$ \\ Linda D'Amore'
}

'Department of Abdominal Wall Surgery, University Hospital (C/O Instituto Clinica Chirugica II), Rome, Italy; ${ }^{2}$ Visceral Surgery, Centre Hospitalier Emile Mayrisch, Esch-sur-Alzette, Luxembourg; ${ }^{3}$ Department of Colorectal Surgery, Whipps Cross University Hospital, Leytonstone, UK; ${ }^{4}$ Colorectal Surgery, Torbay Hospital, Torquay, UK; ${ }^{5}$ Clinic for General and Visceral Surgery,

Universitätsklinikum des Saarlandes, Homburg, Germany; ${ }^{6}$ Department of Abdominal Oncology, Istituto Nazionale Tumori, Napoli, Italy; ${ }^{7}$ General Surgery, Humanitas University and IRCCS Humanitas Research Hospital, Milano, Italy; ${ }^{8}$ General Surgery, Algemeen Ziekenhuis Sint LUCAS, Gent, Belgium; ${ }^{9}$ Thoracic Surgery, CHU Ambroise Pare, Mons, Belgium; ${ }^{10}$ Colorectal Surgery, Royal Victoria Infirmary, Newcastle Upon Tyne, UK

Correspondence: Francesco Gossetti Department of Abdominal Wall Surgery, University Hospital (C/O Instituto Clinica Chirurgica II), Policlinico Umberto I,

Rome, 00162, Italy

Email gossetti@tiscali.it
Purpose: Despite the advancements in the reinforcement and closure techniques available, complex abdominal wall reconstruction (CAWR) remains a challenging surgical undertaking with considerable risk of postoperative complications. Biological meshes were developed that may help to complement standard closure techniques and offer an alternative to synthetic meshes, which carry significant risks with their use in complex cases.

Patients and Methods: A total of 114 patients underwent surgical treatment for CAWR with a Permacol ${ }^{\mathrm{TM}}$ (a biologic surgical implant). The study objective was to evaluate the short-term (6 months), mid-term (12-24 months), and long-term (36 months) clinical outcomes (through 36 months) associated with the use of a biologic surgical implant in these cases.

Results: The cumulative hernia recurrence rate was $18.7 \%$ (17/91) at 24 months and $22.4 \%$ $(19 / 85)$ at 36 months. Twelve (14.1\%) subjects required reoperation for hernia repair within 36 months for repair of recurrent hernias. Between 6- and 36-months post-surgery, patients reported improvement in their Carolina comfort scale (CSS) measures of severity of pain, sensation of mesh, and movement limitations.

Conclusion: A biologic surgical implant can provide long-term benefit to complex abdominal wall repairs in patients staged grade III according to the Ventral Hernia Working Group (VHWG).

Keywords: Permacol, biologic surgical implant, complex abdominal wall repair

\section{Introduction}

Abdominal wall hernias have been reported to occur in as many as $24 \%$ of the patients undergoing abdominal surgery post laparotomy. This number rises when other comorbidities such as prior abdominal surgery, obesity, diabetes, and prior irradiation are factored in. ${ }^{1}$ In these complex situations, it is necessary to carefully consider the surgical approach to ensure optimum recovery for the patient. Despite the numerous advancements in reinforcement and closure techniques available today, complex abdominal wall reconstruction (CAWR) remains a challenging surgical undertaking with considerable postoperative complications. In obese patients with previous repair failures, recurrence rates at 1 year have been reported to be as high as $50 \% .^{1}$ In these patients, each successive repair poses increasing challenges to the surgeon and risks to the patients.

Abdominal wall reconstruction procedures pose challenging wound closure decisions for surgeons. Clearly, beyond identifying those in need of special closure techniques, it is also difficult to decide which closure techniques should be used to 
repair the defects created during surgery. Important considerations regarding the choice for closure techniques for CAWR include the availability of tissue for flap creation as well as the tension at the site of the repair. In cases where there is likely to be insufficient tissue or where there will be a considerable amount of tension, synthetic materials may seem appealing due to their high strength; however, they are considered inappropriate for use in a range of procedures such as those where the mesh may come into contact with the bowel. ${ }^{1-4}$ Abdominal wall reconstruction with synthetic meshes carries a historical hernia recurrence rate of $11 \%$ and overall complication rate of $18-50 \%$, which includes bowel adhesions, fistulization, ulceration, and extrusion, and a $50-90 \%$ risk of infection in contaminated cases. ${ }^{3,4}$ Mathes et al performed a study to compare several reconstructive options for repairing abdominal wall defects which included direct tissue closure, use of synthetic mesh, local advancement or regional flaps, distant flaps, or the combined use of flaps and mesh. Even in smaller defects that were repaired using direct closure, hernia recurrence rates were still $27 \%{ }^{3}$

To date, biological meshes have been developed that may help to complement standard closure techniques and offer an alternative to synthetic meshes that carry significant risks with their use in complex cases. These biological materials are particularly valuable in situations where synthetic meshes are inappropriate for use due to contamination, existing infections, wound dehiscence, unstable wound coverage, diabetes, obesity, patient smoking history, or immunocompromisation. ${ }^{2,4}$ The reported incidence of severe biological mesh-related complications is comparable to, or less than, that of synthetic mesh repairs in CAWR, and the long-term success rates of biological vs synthetic meshes appear to be equivalent. ${ }^{2,5-7}$ Since they can be vascularized rapidly, it is thought that biological meshes could be particularly beneficial where the incidence of fistula and wound infection is high, such as when preoperative chemo-radiation has been used. ${ }^{2,5}$

The present study was undertaken to evaluate shortterm (6 months), mid-term (12-24 months), and long-term (36 months) post-surgical data on Permacol' ${ }^{\mathrm{TM}}$ (a biologic surgical implant) following complex abdominal wall (CAWR) repair.

\section{Materials and Methods}

Between February 2011 and October 2013, patients underwent surgical treatment for complex abdominal wall repair with a biologic surgical implant. The study objective was to evaluate short-term, mid-term, and long-term clinical outcomes associated with the use of a biologic surgical implant in the treatment of complex abdominal wall defects through 36 months ( 3 years) postoperatively. The study was completed in November 2016 and is registered publicly at ClinicalTrials.gov (NCT01268514).

\section{Test Device}

Permacol $^{\mathrm{TM}}$ (a biologic surgical implant) is a sheet of acellular porcine dermal collagen. Permacol ${ }^{\mathrm{TM}}$ is a patented product that is processed to remove non-collagenous and cellular components and is additionally cross linked to enhance durability.

\section{Participants}

Patients $\geq 18$ years of age who required CAWR using a biologic surgical implant were consented if they met eligibility criteria via a screening/baseline visit. A CAWR was defined as infected, contaminated, clean contaminated, or clean with a history of infected or contaminated field, atypical hernia location, loss of domain, fascial dehiscence, and/or requiring abdominal wall mobilization for wound closure. Patients included in the study were 1) subjects who required complex abdominal repair using a biologic surgical implant, 2) were 18 years of age or older, 3) were male or female, and 4) who were willing and able to adhere to protocol requirements and provide written informed consent Exclusion criteria included the following: 1) patients who were pregnant; 2) patients with a medical condition that in the opinion of the investigator may have precluded participation (eg, Ehlers Danlos Syndrome) or interfered with completion of study follow-up; 3) patients could not participate in any other clinical study that clinically interfered with this study while enrolled; 4) concomitant use of a synthetic or another biologic mesh; 5) patients who required use of a biologic surgical implant as only temporary closure with planned reoperation, or a biologic surgical implant used as a temporary dressing in an open abdomen; (6) Patients who had severe systemic sepsis at the time of a biologic surgical implant implantation. Severe sepsis is defined as sepsis with organ dysfunction, hypoperfusion, or hypotension; (7) Patients with ongoing necrotizing pancreatitis; 8) Patients who required use of a biologic surgical implant in parastomal hernia repair alone, where there is no other anterior wall repair; 9) Patients who required prophylactic use of a biologic surgical implant in the formation of stoma with anterior wall repair; and 10) 
a biologic surgical implant used in pelvic floor reconstruction. The trial protocol was approved at each institution's ethics committee (see Supplementary Materials) in accordance with the Helsinki declaration, and all patients provided written informed consent to participate.

\section{Trial Design and Endpoints}

This was a prospective, observational, multicenter study following CAWR (including abdominal wall defects and fascial dehiscence). The observational design of this study intentionally placed no restrictions on procedural technique to ensure generalization and real-world results. Postoperative, assessments were performed at 1 month, 6 months, 12 months, 24 months, and 36 months.

The primary endpoint was to assess the hernia recurrence rate at 36 months post-surgery. Hernia recurrence is defined as a protrusion through the abdominal repair field following assessment by the operating surgeon or equivalent by training and requiring surgical intervention. Secondary endpoints were 1) to characterize short-term and mid-term outcomes within 24 months post-surgery of the proportion of subjects who undergo reoperation for hernia or hernia recurrence; 2) to assess quality of life by Carolinas Comfort Scale at 6 months, 12 months, 24 months, and 36 months (The Carolina comfort scale (CSS) is a Likert-type questionnaire that measures severity of pain, sensation of mesh, and movement limitations ranging from 0 (no symptoms) to 5 (disabling symptoms)); 3) to assess patient satisfaction questionnaire at 6 months, 12 months, 24 months, and 36 months; and 4) for study personnel to assess subjects incidence of postoperative complications, specifically: wound infection, seroma, hematoma, wound dehiscence, and fistula.

\section{Statistics}

Statistical analysis was performed on the per-protocol population (excluding missing data and taking into account patients with at least the 6 months postoperative visit/assessment performed) and consisted of descriptive analysis. Qualitative variables were described by their absolute and relative (\%) frequencies of each class or value, and by two-tailed $95 \%$ confidence intervals. Quantitative variables were described by their mean, standard deviation (SD), extreme values (minimum and maximum values), and number of missing data. Statistical analysis was performed under SAS Version 9.2 and Minitab Version 15.0 using significance of 5\%.

\section{Results}

\section{Patient Disposition and Demographics}

Between February 2011 and December 2016, 122 patients were consented for the study at 12 sites in 5 European countries. Eight patients were screen failures with a final 114 patients included in the full analysis set. Of these 114 patients, 111 (97.4\%) were incisional/abdominal wall cases while $3(2.6 \%)$ were primary hernias. Seventy-three $(64.0 \%)$ patients completed the study while $41(36.0 \%)$ withdrew for reasons including adverse events, lost to follow-up, death, and withdrawal by patient (Figure 1). Patients had a mean age of $60.8 \pm 12.2$ [29-87] years and a mean BMI of $31.2 \pm$ $6.0[18.7-45.4] \mathrm{kg} / \mathrm{m}^{2}$ of which $63(55.3 \%)$ patients had a BMI $\geq 30 \mathrm{~kg} / \mathrm{m}^{2}$ (Table 1). Additionally, 68 (59.7\%) patients were current or past smokers.

\section{Surgical Details}

Most patients underwent surgery with a primary indication of incisional hernias (92.1\%, Table 2). Implant placement

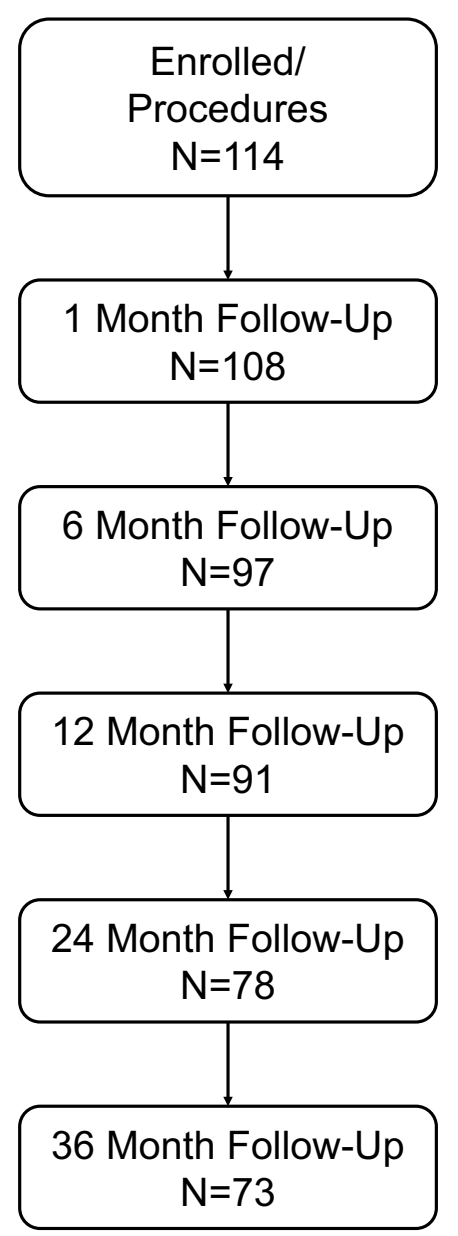

Figure I Study enrollment and follow-up. 
Table I Patient Disposition and Baseline Demographics

\begin{tabular}{|c|c|}
\hline \multicolumn{2}{|l|}{ Patient Dispositions } \\
\hline Consented subjects $^{\mathrm{a}}$ & 122 \\
\hline Screen failures & 8 \\
\hline Full analysis set ${ }^{\mathrm{b}}$ & 114 \\
\hline Per protocol analysis set & 91 \\
\hline Completed study & $73(64.0 \%)$ \\
\hline Withdraw from the study & $41(36.0 \%)$ \\
\hline Primary reason for withdrawal & \\
\hline Adverse event & $20(\mid 7.5 \%)$ \\
\hline Lost to follow-up & $5(4.4 \%)$ \\
\hline Death & $8(7.0 \%)$ \\
\hline Withdrawal by subject & $3(2.6 \%)$ \\
\hline Other & $5(4.4 \%)$ \\
\hline $\begin{array}{l}\text { Patient Baseline } \\
\text { Demographics }\end{array}$ & Full analysis set $(\mathrm{N}=\mathrm{I} \mid \mathrm{4}$ subjects $)$ \\
\hline Age (years), mean $\pm S D$ & $60.8(12.2)$ \\
\hline Female, n (\%) & $47(4 I .2)$ \\
\hline Male, n (\%) & $67(58.8)$ \\
\hline BMI $\left(\mathrm{kg} / \mathrm{m}^{2}\right)$, mean $\pm \mathrm{SD}$ [range] & $31.2 \pm 6.0[18.7-45.4]$ \\
\hline$<30, \mathrm{n}(\%)$ & $5 \mathrm{I}(44.7)$ \\
\hline$\geq 30, \mathrm{n}(\%)$ & $63(55.3)$ \\
\hline Smoking status & Full analysis set $(\mathrm{N}=\mathrm{I} \mid 4$ subjects) \\
\hline Current smoker & $23(20.2 \%)$ \\
\hline Past smoker & 45 (39.5\%) \\
\hline Non-smoker & 46 (40.4\%) \\
\hline Duration of smoking (years) & $28.2 \pm 11.6[2-54]$ \\
\hline
\end{tabular}

Notes: ${ }^{a}$ One patient was included in the Safety analysis set but excluded from the FAS (Performance analysis), as they received both Prolene mesh and Permacol. ${ }^{b}$ Percentages and means are based on the number of FAS subjects.

techniques and fixation methods varied, but the majority of surgeries used intra peritoneal onlay $(\mathrm{n}=89,78.1 \%)$, and either non-resorbable sutures $(\mathrm{n}=50,43.9 \%)$ or slowly resorbable sutures $(\mathrm{n}=59,51.8 \%)$ (Table 2). Hernias had a mean length of $15.1 \pm 8.8 \mathrm{~cm}[2-35 \mathrm{~cm}]$ and a mean width of $11.6 \pm 6.9 \mathrm{~cm}[3-35 \mathrm{~cm}]$. The majority of procedures used either the $20 \times 30 \mathrm{~cm}(\mathrm{n}=32,28.1 \%)$ or $18 \times 28 \mathrm{~cm}(\mathrm{n}=30,26.3 \%)$ implants for the first use. In addition, six procedures used a second implant of varying sizes and no procedures used a third implant (Table 2). The total mean implant area for the procedures was $460.7 \pm$ $213.7 \mathrm{~cm}$ with a minimum of $100 \mathrm{~cm}$ and a maximum of $1100 \mathrm{~cm}$. In total, facial closure was achieved on 101 $(88.6 \%)$ of the patients. No device malfunctions were reported during the study (Table 2). Additionally, although 42 patients had Ventral Hernia Working Group (VHWG) grade III or IV, additional patients with VHWG grade I and II were included due to the presence of various comorbidities.

\section{Hernia Recurrence and Adverse Events}

The cumulative hernia incidence was $18.7 \%$ (17/91) at 24 months and $22.4 \%(19 / 85)$ at 36 months (Table 3). Twelve (14.1\%) subjects required reoperation within 36 months for repair of recurrent hernias. Total peri- and postoperative complications included $13(11.3 \%)$ wound dehiscences, 11 (9.6\%) wound infections, 11 (9.6\%) seromas, and $4(3.5 \%)$ hematomas (Table 3$)$. Additionally, a single case $(0.9 \%)$ of stoma site pain was reported.

\section{Quality of Life}

Between 6- and 36-months post-surgery, patients showed improvement in all three scores. In particular, significant improvement $(p=0.0036)$ was seen in the reduction of pain at 24 months (Table 4).

\section{Discussion}

The study was initiated in 2011 and completed in 2016. Although the study design may now appear outdated, it was based on criteria and methods adopted in that period and as a result, demographics were reported as precisely as possible. Additionally, while the prospective 3-year follow-up was standard at the time of study design, newer studies now use 5-year follow-up to assess longer-term outcomes and late complications.

The hernia recurrence rate identified as the primary endpoint of this study appears to be aligned with other literature. ${ }^{3,4}$ However, although recurrence remains a main problem in abdominal wall repair, it is no longer the only aspect to be considered. Many other features have been demonstrated to be critical when a biological implant could be used, mainly indications and costs. In 2015, the Canadian Agency for Drug and Technologies in Health asked four questions to consider about this issue: 1) What is the clinical effectiveness of biological implants? 2) What is their cost-effectiveness? 3) What are the evidence-based guidelines regarding appropriate clinical indications? And 4) What are the evidence-based guidelines regarding their use?

These questions are still largely unresolved and there is insufficient high-quality evidence to suggest the optimal 
Table 2 Surgical Details

\begin{tabular}{|c|c|c|c|}
\hline & $\mathbf{N}(\%)$ & & $\mathbf{N}(\%)$ \\
\hline Primary indication for surgery & 114 & Number of Permacol implants used & 114 \\
\hline Abdominal wall repair & $6(5.3)$ & & \\
\hline Primary hernia & $3(2.6)$ & First piece used & 114 \\
\hline Incisional hernia & $105(92.1)$ & $10 \times 10 \mathrm{~cm}$ & $7(6.1)$ \\
\hline \multirow[t]{2}{*}{ Missing } & I & $10 \times 15 \mathrm{~cm}$ & $15(13.2)$ \\
\hline & & $15 \times 20 \mathrm{~cm}$ & $21(18.4)$ \\
\hline Implant placement technique & 114 & $18 \times 28 \mathrm{~cm}$ & $30(26.3)$ \\
\hline Intra peritoneal onlay & $89(78.1)$ & $20 \times 30 \mathrm{~cm}$ & $32(28.1)$ \\
\hline Sublay & $19(16.7)$ & $20 \times 40 \mathrm{~cm}$ & $9(7.9)$ \\
\hline Inlay & $3(2.6)$ & Missing & 0 \\
\hline Onlay & $2(1.8)$ & & \\
\hline Other: & I (0.9) & Second piece used & 6 \\
\hline "Sublay+intraperitoneal position" & $\mathrm{I}(0.9)$ & $5 \times 10 \mathrm{~cm}$ & I (16.7) \\
\hline \multirow[t]{2}{*}{ Missing } & 0 & $10 \times 15 \mathrm{~cm}$ & $2(33.3)$ \\
\hline & & $15 \times 20 \mathrm{~cm}$ & I (16.7) \\
\hline Fixation & 114 & $18 \times 28 \mathrm{~cm}$ & I (16.7) \\
\hline Non-resorbable sutures & $50(43.9)$ & $20 \times 30 \mathrm{~cm}$ & I (I6.7) \\
\hline Slowly resorbable sutures (PDS, MAXON) & $59(51.8)$ & & \\
\hline Resorbable sutures & $6(5.3)$ & Third piece used & 0 \\
\hline Resorbable tacks & $2(1.8)$ & & \\
\hline Non-resorbable tacks & I (0.9) & Total implant area $\left(\mathrm{cm}^{2}\right)$ & 114 \\
\hline Glue & $2(1.8)$ & Missing & 0 \\
\hline Other & 0 & Mean (SD, SE) & $460.7(2 \mid 3.7)$ \\
\hline \multirow[t]{3}{*}{ Missing } & 0 & Median & 504.0 \\
\hline & & Min/Max & $100-1100$ \\
\hline & & Device malfunctions & 0 \\
\hline
\end{tabular}

indications for the use of biological meshes in CAWR as confirmed in a recent review performed by Kockerling. ${ }^{8}$

In the present study, the majority of patients had a VHWG grade I and II (72/114) and this contrasts with current indications that restrict the use of biologics to VHWG grade III. It is well known that the VHWG grade represents an independent risk factor for recurrence and complications. In fact, according to Slater, the number of comorbidities seems to be strictly related to postoperative adverse events. ${ }^{9}$ As a result, the best results should be achieved with a low-grade VHWG. In the present study, however, it was observed that recurrence rates reported by sites using a biologic surgical implant in grade I and II cases were higher than expected, while those sites utilizing the implant in grade III cases were lower than expected (Table 5). This higher recurrence rate in grade I and II can be explained by the inappropriate techniques used, including fixation and limited mesh overlap. This seems to confirm that the implant of a biologic surgical implant in potentially contaminated fields is a correct indication which can lead to satisfying results. In grade IV, the use of any prosthetic material is contraindicated and a downstaging to grade III, according to accepted medical practice, eventually using negative pressure wound therapy (NPWT) is advisable. In grade IV cases, a biologic surgical implant cross-linked implant presents a high risk of infection that may lead to weakening or breakdown and the possibility of the mesh to be removed, according to data from literature. ${ }^{10}$ In the same situation, non-crosslinked implants do not need to be removed because they are destroyed by infection leading to subsequent high risk of hernia recurrence.

The high number of sites participating in the study reflects the relatively low number of subjects enrolled by each individual site (range 2-27). Moreover, numerous surgeons with different degrees of skill and experience in abdominal wall repair took part in the study, so that a suitable number of cases could be reached. Indications, operative techniques, and mesh implant sites varied across clinical sites and likely impacted patient outcomes, especially concerning operative technique and mesh site of implant, that play a primary role in the outcome of the 
Table 3 Hernia Recurrence and Adverse Events

\begin{tabular}{|l|c|c|c|c|}
\hline & N & Cumulative Hernia Recurrence & Cumulative Surgeries to Correct Hernia Recurrences $^{\mathbf{a}}$ & Missing \\
\hline I-month post-surgery & 110 & $3(2.7 \%)$ & $2(1.8 \%)$ & 4 \\
6-month post-surgery & 106 & $6(5.7 \%)$ & $4(3.8 \%)$ & 8 \\
I2-month post-surgery & 102 & $12(11.8 \%)$ & $9(8.8 \%)$ & 12 \\
24-month post-surgery & 91 & $17(18.7 \%)$ & $12(13.2 \%)$ & 23 \\
36-month post-surgery & 85 & $19(22.4 \%)$ & $12(14.1 \%)$ & 29 \\
\hline
\end{tabular}

\begin{tabular}{|c|c|}
\hline Adverse Event ${ }^{\mathrm{b}}$ & Subjects by AE Category ( $N=$ I I 5 Subjects), $N(\%)$ \\
\hline Subjects with at least I device-related $\mathrm{AE}$ & $82(71.3)$ \\
\hline Wound dehiscence & $13(11.3)$ \\
\hline Wound infection & II (9.6) \\
\hline Seroma & II (9.6) \\
\hline Abdominal hernia & $10(8.7)$ \\
\hline Abdominal pain & $8(7.0)$ \\
\hline Implant site extravasation & $7(6.1)$ \\
\hline Incisional hernia & $7(6.1)$ \\
\hline Intestinal obstruction & $5(4.3)$ \\
\hline Pulmonary embolism & $4(3.5)$ \\
\hline Hematoma & $4(3.5)$ \\
\hline Myocardial infarction & $3(2.6)$ \\
\hline Impaired healing & $3(2.6)$ \\
\hline Postoperative wound infection & $3(2.6)$ \\
\hline Arthralgia & $3(2.6)$ \\
\hline Anaemia & $2(1.7)$ \\
\hline Cardiac failure & $2(1.7)$ \\
\hline Enterocutaneous fistula & $2(1.7)$ \\
\hline lleus & $2(1.7)$ \\
\hline Inguinal hernia & $2(1.7)$ \\
\hline Umbilical hernia & $2(1.7)$ \\
\hline Vomiting & $2(1.7)$ \\
\hline Staphylococcal infection & $2(1.7)$ \\
\hline Abdominal wound dehiscence & $2(1.7)$ \\
\hline Intervertebral disc protrusion & $2(1.7)$ \\
\hline Other ${ }^{c}$ & $64(55.7)$ \\
\hline
\end{tabular}

Notes: ${ }^{a}$ For cumulative corrective surgery, corrective surgery results are based on cumulative hernia $=$ Yes at each assessment (including unscheduled surgery data). ${ }^{\mathrm{b}}$ Each subject is only counted once per system organ class and per preferred term. ${ }^{\mathrm{C}}$ Adverse events with $>$ I subject are individually listed.

Table 4 Carolinas Comfort Scale Scores

\begin{tabular}{|c|c|c|c|}
\hline Mean (SD) & $\begin{array}{c}\text { Sensation } \\
\text { of Mesh }\end{array}$ & Pain & $\begin{array}{c}\text { Movement } \\
\text { Limitations }\end{array}$ \\
\hline 6-month post-surgery $(\mathrm{N}=84)$ & $3.6(6.0)$ & $4.2(6.9)$ & $3.7(6.3)$ \\
I2-month post-surgery $(\mathrm{N}=80)$ & $3.11(5.1)$ & $2.6(4.9)$ & $1.9(4.2)$ \\
24-month post-surgery $(\mathrm{N}=70)$ & $2.5(5.5)$ & $2.5(7.0)^{*}$ & $2.6(6.2)$ \\
36-month post-surgery $(\mathrm{N}=65)$ & $2.6(6.0)$ & $2.0(5.6)$ & $2.4(6.0)$ \\
Unscheduled surgery $(\mathrm{N}=3)$ & $1.0(1.7)$ & $3.0(5.2)$ & $3.0(5.2)$ \\
\hline
\end{tabular}

Note: ${ }^{*} p=0.0036$ compared to 6-month scores.

patient. A comment by Negro et al regarding the publication by Abdelfatah et al, underlined the key role of correct indications and, overall, on a proper surgical technique for successful CAWR with a biologic surgical implant, after
Table 5 Hernia Recurrences by VHWG Wound Grade

\begin{tabular}{|l|l|l|}
\hline VHWG Wound Grade & N & Hernia Recurrence, N (\%) \\
\hline Grade I & 16 & $2(12.5)$ \\
Grade II & 56 & $10(17.9)$ \\
Grade III & 34 & $4(11.8)$ \\
Grade IV & 8 & $3(37.5)$ \\
\hline
\end{tabular}

Abbreviations: BMI, body mass index; CAWR, complex abdominal wall reconstruction; CSS, Carolina comfort scale; NPWT, negative pressure wound therapy; SD, standard deviation; SSI, surgical site infection; SSO, surgical site occurrence; VHWG, Ventral Hernia Working Group.

Abdelfatah's series reported a recurrence rate of $13 \%$ versus $66 \%$ with similar incidence of surgical site infection (SSI). ${ }^{10,11}$ 
With regards to the surgical procedures, the technique of placement of the implant seems to be correct in $94.8 \%$ of cases, with $78.1 \%$ of intraperitoneal "bridge" and $16.7 \%$ of retromuscular sublay placement. Although closing the defect where possible is highly recommended, in cases when closure is not achievable, it is correct, according to Italian Guidelines and the Italian Biological Prosthesis Working Group, to use a cross-linked implant in a "bridge" position while a non-cross-linked implant would likely assure failure. ${ }^{12}$ Alternatively, in $5.2 \%$ of the cases, an incorrect technique with onlay or inlay positioning is reported. Both these techniques, in fact, had been progressively dismissed due to the high recurrence rate and the high risk of SSO and SSI, confirmed by our personal experience also. In the site with the highest recurrence rate, many different techniques were performed, emphasizing how an unstandardized procedure leads to disappointing results; however, these results may more accurately mimic what health care providers see in real-world cases.

Regarding mesh fixation and size of implant, $46.6 \%$ of surgeons used non-resorbable fixations (43.9\%, nonresorbable sutures, $0.9 \%$ non-resorbable tacks, $1.8 \%$ glue) while $51.8 \%$ utilized unsuitable fixation methods, as slowly resorbable sutures (PDS, Maxon), resorbable tacks and glue, that reabsorb before the remodeling of the cross-linked implants has been completed. Additionally, in six patients, two implants were sutured together. This increases the risk of recurrence but underlines the need of larger prostheses. Of note, in $19.3 \%$ of the cases, the biological implant measured only $150 \mathrm{~cm}^{2}$. Given the relatively small size of these meshes, it should be meaningful to understand if the extent of the mesh was suitable to properly cover the defect. Taking into account hernia recurrence and follow-up, the overall trend is aligned with previous data from literature. ${ }^{12}$

Results from the present study are limited by the relatively low number of patients enrolled by each site, the variety of indications for the use of biological implants, and the lack of standardization of operative techniques across sites. Moreover, although the 3-year follow-up could be considered adequate at the time the study was initiated, an extension to at least 5 years would have been preferable.

\section{Conclusion}

The hernia recurrence rate of $22.4 \%$ found in this 3 -year follow-up study is similar to that found in current literature and suggests that the use of a biologic surgical implant can provide long-term benefit to CAWR in patients staged grade III according to VHWG.

\section{Acknowledgments}

This study was sponsored by Medtronic. Nicholas Paquette PhD (Medtronic) provided medical writing assistance under the authors' direction and based on content and conclusions developed by the authors. The patient datasets generated and analyzed during the current study are not publicly available due to restrictions that apply to the availability of these data. Data are however available from F. Gossetti (gossetti@tiscali.it) upon reasonable request and with permission of Medtronic.

\section{Disclosure}

$\mathrm{Mr}$ Pasquale Giordano reports personal fees from Medtronic, outside the submitted work. Professor Marco Montorsi reports personal fees from Baxter, outside the submitted work. Dr Ben Griffiths reports non-financial support and personal fees from Medtronic. The authors report no other conflicts of interest in this work.

\section{References}

1. Davison SP, Parikh PM, Jacobson JM, Iorio ML, Kalan M. A "buttressed mesh" technique for fascial closure in complex abdominal wall reconstruction. Ann Plast Surg. 2009;62(3):284-289. PubMed PMID: 19240526. doi:10.1097/SAP.0b013e31817e9c6d

2. Baillie DR, Stawicki P, Eustance N, Warsaw D, Desai D. Use of human and porcine dermal-derived bioprostheses in complex abdominal wall reconstructions: a literature review and case. Ostomy Wound Manag. 2007;53(5):30-37.

3. Mathes SJ, Steinwald PM, Foster RD, Hoffman WY, Anthony JP. Complex abdominal wall reconstruction: a comparison of flap and mesh closure. Ann Surg. 2000;232(4):586-596. PubMed PMID: 10998657; PubMed Central PMCID: PMCPMC1421191. doi:10.10 97/00000658-200010000-00014

4. Rodriguez ED, Bluebond-Langner R, Silverman RP, et al. Abdominal wall reconstruction following severe loss of domain: the $\mathrm{R}$ Adams Cowley Shock Trauma Center algorithm. Plast Reconstr Surg. 2007;120(3):669-680. PubMed PMID: 17700118. doi:10.1097/01. prs.0000270303.44219.76

5. Butler CE, Langstein HN, Kronowitz SJ. Pelvic, abdominal, and chest wall reconstruction with AlloDerm in patients at increased risk for mesh-related complications. Plast Reconstr Surg. 2005;116(5):126375; discussion 76-7. PubMed PMID: 16217466. doi:10.1097/01. prs.0000181692.71901.bd

6. Catena F, Ansaloni L, Gazzotti F, et al. Use of porcine dermal collagen graft (Permacol) for hernia repair in contaminated fields. Hernia. 2007;11(1):57-60. doi:10.1007/s10029-006-0171-6

7. Franklin ME, Gonzalez JJ, Glass JL. Use of porcine small intestinal submucosa as a prosthetic device for laparoscopic repair of hernias in contaminated fields: 2-year follow-up. Hernia. 2004;8(3):186-189. doi:10.1007/s10029-004-0208-7

8. Köckerling F, Alam NN, Antoniou SA, et al. What is the evidence for the use of biologic or biosynthetic meshes in abdominal wall reconstruction? Hernia. 2018;22(2):249-269. doi:10.1007/s10029018-1735-y 
9. Slater NJ, Montgomery A, Berrevoet F, et al. Criteria for definition of a complex abdominal wall hernia. Hernia. 2014;18(1):7-17. PubMed PMID: 24150721. doi:10.1007/s10029-013-1168-6

10. Abdelfatah MM, Rostambeigi N, Podgaetz E, Sarr MG. Long-term outcomes ( $>5$-year follow-up) with porcine acellular dermal matrix (Permacol) in incisional hernias at risk for infection. Hernia. 2015;19 (1):135-140. PubMed PMID: 24129420. doi:10.1007/s10029-013$1165-9$

11. Negro P, D'Amore L, Ceci F, Gossetti F. Comment to "Long-term outcomes ( $>5$ year follow-up) with porcine acellular dermal matrix (Permacol) in incisional hernias at risk for infection" by Abdelfatah MM, Rostambeigi N, Podgaetz E, Sarr MG (DOI 10.1007/s10029013-1165-9). Hernia. 2015;19(6):1023-1024. PubMed PMID: 26169535. doi:10.1007/s10029-015-1406-1
12. Flum DR, Horvath K, Koepsell T. Have outcomes of incisional hernia repair improved with time? A population-based analysis. Ann Surg. 2003;237(1):129-135. PubMed PMID: 12496540. doi:10.1097/ 00000658-200301000-00018
Medical Devices: Evidence and Research

\section{Publish your work in this journal}

Medical Devices: Evidence and Research is an international, peerreviewed, open access journal that focuses on the evidence, technology, research, and expert opinion supporting the use and application of medical devices in the diagnosis, monitoring, treatment and management of clinical conditions and physiological processes. The identification of novel devices and optimal use of existing devices

\section{Dovepress}

which will lead to improved clinical outcomes and more effective patient management and safety is a key feature of the journal. The manuscript management system is completely online and includes a very quick and fair peer-review system. Visit http:// www.dovepress.com/testimonials.php to read real quotes from published authors.

Submit your manuscript here: https://www.dovepress.com/medical-devices-evidence-and-research-journal 